$$
\angle A-S \cup B-97-55
$$

SUMNER ASSOCIATES

100 Cienega, Suite D

Santa $\mathrm{Fe}$, New Mexico 87501

Telephone: 505 984-8041 FAX 505 984-3251
RECEIVED

FFR 171998

OSTI

FINAL REPORT

0412J0004-3Y

TASK 57

October 3, 1997

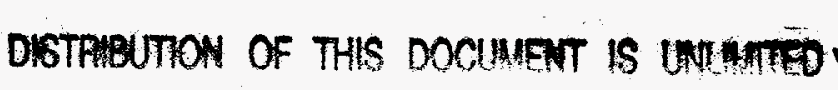




\section{DISCLAIMER}

This report was prepared as an account of work sponsored by an agency of the United States Government. Neither the United States Government nor any agency thereof, nor any of their employees, makes any warranty, express or implied, or assumes any legal liability or responsibility for the accuracy, completeness, or usefulness of any information, apparatus, product, or process disclosed, or represents that its use would not infringe privately owned rights. Reference herein to any specific commercial product, process, or service by trade name, trademark, manufacturer, or otherwise does not necessarily constitute or imply its endorsement, recommendation, or favoring by the United States Government or any agency thereof. The views and opinions of authors expressed herein do not necessarily state or reflect those of the United States Government or any agency thereof. 


\section{DISCLAIMER}

Portions of this document may be illegible electronic image products. Images are produced from the best available original document. 


\title{
MLNSC Instrument Design and Simulation Package \\ Task Order 57 (modified) \\ Final Report, September 30, 1997
}

\author{
Philip A. Seeger \\ Sumner Associates
}

The objectives of this task as described in Statement of Work have been met and the documents required as Deliverables have been prepared and submitted to the requester.

Specifically, a document titled "The MCLIB Library: Monte Carlo Simulation of Neutron Scattering Instruments," revised Sept. 23, 1997, includes documentation of new standards, code revisions and additions, and some improved efficiency due to improved optimization strategies. The procedures for user implementation of new optical devices, and information on using the package and reading and viewing the output have also been included. The full text of this document ( 66 pages) may be obtained by anonymous ftp from snow.lansce.lanl.gov/pub/mclib/document/. Formats available include Microsoft Word 6.0 (mclib.doc), Adobe portable document (mclib.pdf), or ASCII text without figures (mclib.tat).

Second, a new document entitled "MCLIB Element Definitions and Help" was written and revised through the duration of the task, to supply the needed input to group CIC-15 for the purpose of integrating the MCLIB package with a web-based user interface. The document version of Sept. 30, 1997, includes definitions of the 18 beam-line devices considered most essential for the early use of the interface. These have been implemented by $\mathrm{CIC}-15$, and the user interface is now functional. In addition to the mathematical algorithms, on-line help messages are provided. The full text of this document (24 pages) may be obtained by anonymous ftp from snow. lansce.lanl.gov/pub/mclib/document/. Formats available include Microsoft Word 6.0 (ElmntDef.doc) or Adobe portable document (ElmntDef.pdf), and the help messages have also been converted to html (ElmntHelp.html) for direct inclusion in the interface. Priorities have been discussed for addition of more element types.

Finally, an application of the package was presented and a (successful) demonstration of the new user interface was given at a workshop at Argonne National Laboratory, August 24-26, 1997, as described in the modification to the Statement of Work. Response of the other workshop participants seemed quite favorable. An indication of the interest is that another workshop, devoted expressly to defining code standards, has been scheduled at Argonne in November. We believe that this code package is the only one available which meets the stated goals in the call to the workshop, and are thus hopeful that full acceptance and participation in development of the code by the neutron user community may be achieved. A copy of the contribution to the proceedings of the August workshop is attached to this report. 


\title{
USING THE MCLIB LIBRARY: SMALL-ANGLE INSTRUMENT WITH FOCUSING-MIRROR COLLIMATION
}

\author{
PHILIP A. SEEGER, Consultant \\ Manuel Lujan Jr. Neutron Scattering Center and Sumner Associates \\ Los Alamos National Laboratory \\ Los Alamos, NM 87545 USA
}

\begin{abstract}
Focusing mirrors in 1 or 2 dimensions can be used to increase the intensity in a small-angle instrument while maintaining high resolution. Using the recently added toroidal mirror region type in MCLIB (Monte Carlo library for neutron instrument design), we compare five mirror shapes: the ideal ellipsoid, the tangent toroid, 8 tangent toroidal segments, 8 tangential cylindrical segments, and 20 tangential cylindrical segments. A small-angle scattering instrument on a spallation source was simulated to study resolution. Comparisons show that the ellipsoid provides superior resolution, but that the 8 tangential toroids are a good compromise. A resolution of $0.00056 A^{-1}$ ( $\mathrm{ms}$ ), or $\pm 11 \%$ at $Q=0.005 \AA^{-1}$, was achieved in the simulation using neutrons in the wavelength range $4-15 \AA$. The count rate is high because the full area of the moderator is viewed. The latest release of the MCLIB source code and documentation may be obtained by anonymous ftp from ftp://azoth.lanscelanl.gov/pub/mclib/. Work is also continuing on a friendly web-based user interface, which can be found at http://bayberry.lanl.gov/lansce/Welcome.himl. User input is being sought for improvements to the library and to the interface.
\end{abstract}

\section{Introduction}

Focusing mirrors in 1 or 2 dimensions can be used to increase the intensity in a small-angle instrument while maintaining high resolution (e.g., better than $\mathrm{Q}_{\min }$ $\left.=0.001 \AA^{-1}\right)^{1,2}$ A small-angle scattering instrument on a spallation source was simulated with $\mathrm{MCLIB}^{3}$ to study resolution. The authors of ref. 1 used small-angle scattering with one-dimensional focusing as an example, but the same arguments concerning solid-angle matching can be applied in two dimensions using an ellipsoidal rather than cylindrical mirror. Toroids are also considered as approximations to the ellipsoid 2,4 . Mirror shapes to be compared include the ideal ellipsoid, tangential toroidal segments, and tangential cylindrical segments.

\section{The Instrument}

The instrument is shown schematically in Fig. 1. The moderator is coupled liquid $\mathrm{H}_{2}$, as proposed for the upgrade at the Manuel Lujan Jr. Neutron Scattering Center. Three choppers have been included: a TO chopper to remove the high-energy pulse, 
a frame-overlap (tail) chopper, and a frame-definition chopper which allows different wavelength ranges to be selected. In these simulations the range was 3.5$15.0 \AA$, at a $20-\mathrm{Hz}$ pulse rate and full instrument length of $16 \mathrm{~m}$. The $2-\mathrm{mm}$ diameter aperture is at one focus of the ellipsoid and the detector is placed at the other focus. The mirror is $4.00 \mathrm{~m}$ long and $100 \mathrm{~mm}$ wide, with a major axis of $9.142 \mathrm{~m}$ and transverse (minor) axes of $239 \mathrm{~mm}$, inclined $1.5^{\circ}$ upward. The aperture and the detector are each $4.517 \mathrm{~m}$ from the mirror center, and the total bend angle is $3^{\circ}$ (upward to correct for gravity ${ }^{4}$ ). The sample is $54-\mathrm{mm}$ square and is located $2.50 \mathrm{~m}$ in front of the detector. Three "scatterers" were simulated: no sample, to observe the beam spot shape and statistics; a $\delta$-function at $Q=0.005$ $\AA^{-1}$ to compute instrument resolution directly; and hard spheres of radius $1000 \AA$, to illustrate data quality. For the latter two systems, the detector was recorded in 1 -mm square pixels with an rms encoding error of $\pm 0.5 \mathrm{~mm}$.

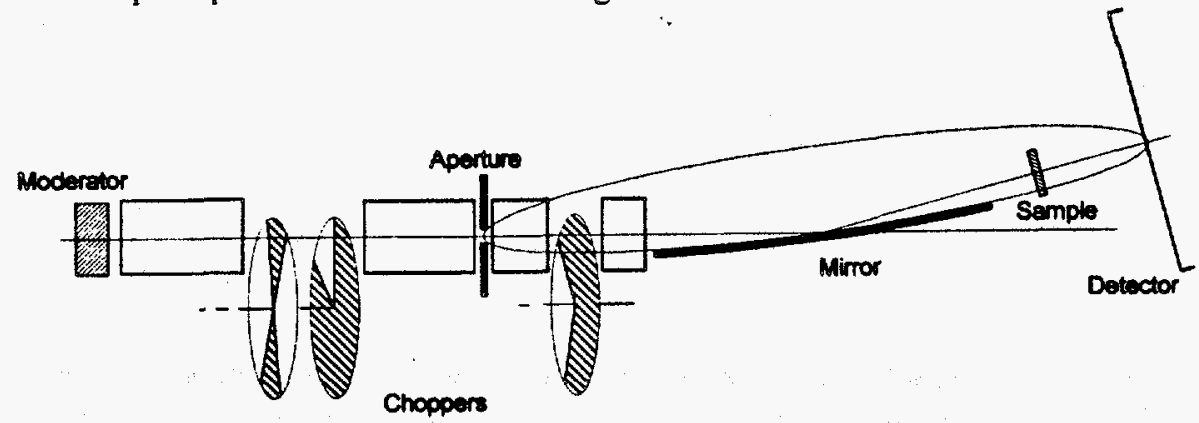

Figure 1. Layout of small-angle scattering instrument with focusing mirror. Transverse dimensions are exaggerated compared to longitudinal.

Five mirror configurations were compared. First is the ellipsoid itself, which was defined as a single surface but may also be simulated as separate segments to study the effects of misalignments. Since toroids may be easier to fabricate, the toroid tangent to the ellipsoid at its center was run next. Then a segmented mirror with $\mathbf{8}$ toroids, independently tangent to segments of the ellipsoid, was considered. To test if the second order bend of the toroids is necessary, a mirror of 8 cylinders was tested. This was so poor an approximation to the ellipsoid that a mirror with 20 cylindrical segments was also computed.

\section{Results}

The results for the beam spot shapes are shown in Fig. 2. Each run represents an integrated proton beam of $6.7 \mathrm{MW}$-s, which was $10^{6}$ detected neutrons in the 


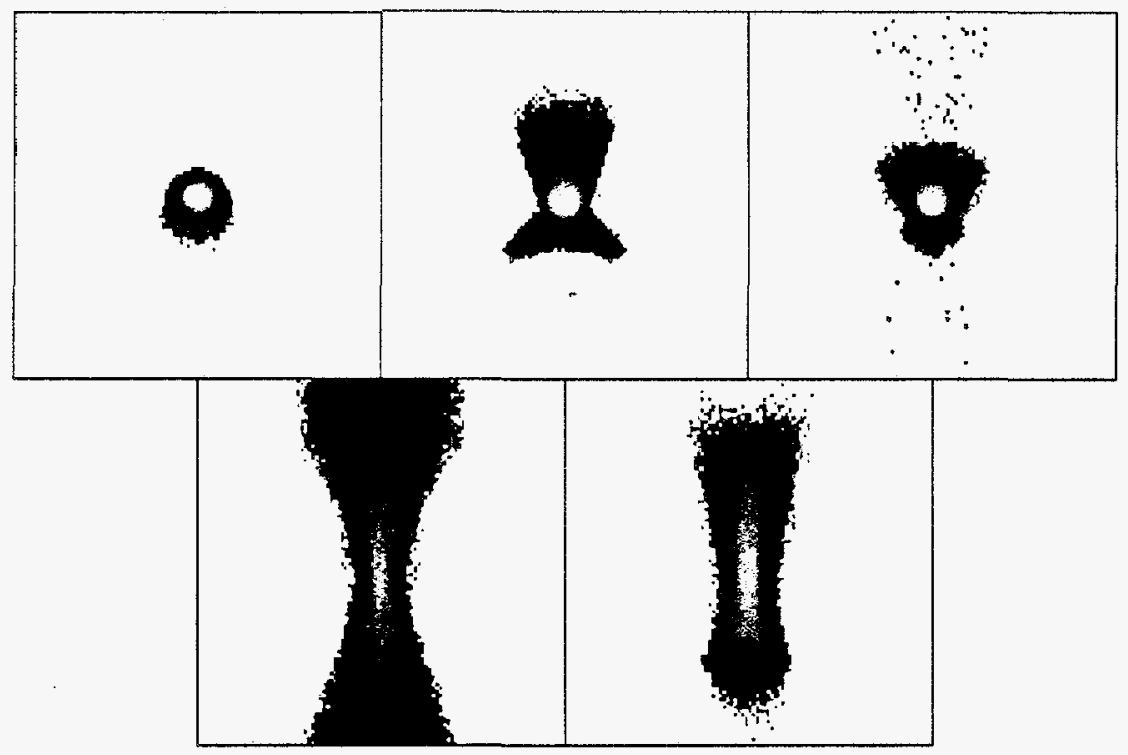

Figure 2. Beam spot profiles. Upper: Ellipsoid, Toroid, 8 Toroids. Lower: 8 Cylinders, 20 Cylinders. Figures are approximately twice actual size. The intensity scale is logarithmic.

ellipsoid case. The rms values of the beam spots and also the resolution at $Q=$ $0.005 \AA^{-1}$ are given in Table 1 , which also has a figure of merit (FOM) based on intensity divided by variance. Comparisons of the resolution and of the data for $\mathrm{R}$ $=1000 \AA$ show that the ellipsoid is clearly superior, but that the 8 tangential toroids are a good compromise (if easier to construct). A resolution of $0.00056 \AA^{-1}$ (rms), or $\pm 11 \%$, was achieved using neutrons in the wavelength range 4-15 $\AA$.

Table 1: Resolution Results at $Q=0.005 \AA^{-1}$

\begin{tabular}{|ccccc|}
\hline Mirror & $\mathrm{rms} \mathrm{X}(\mathrm{mm})$ & $\mathrm{rms} \mathrm{Y}(\mathrm{mm})$ & $\mathrm{rms} \mathrm{Q}\left(\AA^{-1}\right)$ & FOM \\
\hline Ellipsoid & 0.56 & 0.56 & 0.00056 & 3.43 \\
Toroid & 0.80 & 1.34 & 0.00081 & 1.87 \\
8 Toroids & 0.62 & 0.82 & 0.00057 & 3.31 \\
8 Cylinders & 1.16 & 5.72 & 0.00222 & 0.30 \\
20 Cylinders & 0.75 & 3.17 & 0.00115 & 1.08 \\
\hline
\end{tabular}

\section{Obtaining the MCLIB Code}

The latest release of the MCLIB source code and documentation may be obtained 
by anonymous ftp from ftp://azoth.lansce.lanl.gov/pub/mclib/. Work is also continuing on a friendly web-based user interfaces, which can be found at http://baybery.lanl.gov/lansce/Welcome.html. You can get more information about the project by clicking on "Click here for more information" near the bottom of that page. There will be a library of standard elements which you assemble into an instrument. You then download a file containing all of the information in the proper format for input to the program MC_RUN (which you also download), and make the actual Monte Carlo run on your own computer. User input is being sought for improvements to the library and to the interface. Comments and suggestions can be sent to the author by e-mail toPASeeger@aol.com.

\section{Acknowledgements}

This work was supported by the U. S. Department of Energy, Office of Basic Energy Sciences and other Department of Energy programs under contract W7405-ENG-32 to the University of California.

\section{References}

1. H. Maier-Leibnitz and T. Springer, "The use of neutron optical devices on beam-hole experiments," Reactor Science and Technology (J. Nucl. Energy $A / B$ 17) $217-225$ (1963).

2. J. R. D. Copley, "Simulations of neutron focusing with curved mirrors," Rev. Sci. Instrum. 67, 188-194 (1996).

3. P. A. Seeger, "The MCLIB Library: Monte Carlo simulation of neutron scattering instruments," 13th Meeting of the International Collaboration on Advanced Neutron Sources, October 11-14, 1995, Paul Scherrer Institut, Villigen, Switzerland, PSI Proceedings 95-02, pp. 194-212.

4. P. A. Seeger, "The MCLIB Library: new features," Workshop on Methods for Neutron Scattering Instrument Design, September 23-26, 1996, Lawrence Berkeley National Laboratory.

5. T. G. Thelliez, L. L. Daemen, P. A. Seeger, and R. P. Hjelm, Jr., "A userfriendly geometry interface for the Monte Carlo neutron optics code MCLIB," 13th Meeting of the International Collaboration on Advanced Neutron Sources, October 11-14, 1995, Paul Scherrer Institut, Villigen, Switzerland, PSI Proceedings 95-02, pp. 307-311. 